\title{
Retrieval structures and schemata: A brief reply to Ericsson and Kintsch
}

Gobet, F. (2000) Retrieval structures and schemata: A brief reply to Ericsson and Kintsch. British Journal of Psychology, 91, 591-594.

\section{Fernand Gobet}

ESRC Centre for Research in Development, Instruction and Training

School of Psychology

University of Nottingham

Nottingham NG7 2RD

England

Phone: (0115) 9515402

Fax: (0115) 9515324

frg@ psyc.nott.ac.uk

Running head: Retrieval structures and schemata 


\section{Retrieval structures and schemata:}

\section{A brief reply to Ericsson and Kintsch}

In their commentary, Ericsson and Kintsch (henceforth EK) address several important issues. While I am more convinced than they are about the substantial similarities shared by our two approaches, and hence their comparability, this short reply will mostly limit itself to matters of disagreement.

\section{Comparison, not translation}

EK emphasise the differences between long-term working memory (LTWM) and EPAM/CHREST. However, I believe that the two approaches also share many important features, both theoretical and methodological, including: rapid encoding into LTM; the role given to the environment; gradual learning and refinement of knowledge; the importance of individual differences; the importance of representative tasks to capture expertise; and a penchant for using single-subject designs with high density data.

In spite of these similarities, there obviously remain some significant differences, particularly our views about the type of theory that should be preferred. In particular, EK propose that a general theory of expertise is more likely to be at the level of LTWM mechanisms, and not at the level of the elementary processes incorporated in EPAM/CHREST. The two levels are not incompatible, however. Although EPAM/CHREST incorporates low-level information-processing mechanisms, it can also be used to offer explanations at a higher level of abstraction, using concepts such as "chunks" or "schemata" — of course with some loss of precision.

My critique of LTWM had two goals: (a) to evaluate LTWM's account of the empirical data; and (b) to compare how LTWM and EPAM/CHREST construe the concept of retrieval structure. My goal was emphatically not to translate or specify LTWM within EPAM/CHREST, as EK imply several times. Moreover, although EK (p. 3) state that "Gobet's criticisms of LTWM failed to recognize the basic differences between the retrieval structures [of LTWM and EPAM-IV]", I actually listed five key differences (most of them relating to retrieval structures) between LTWM and EPAMIV.

\section{Scope of LTWM and TT}

This debate has been useful in clarifying at least two points. First, EK (p. 12) make it clear that Ericsson and Kintsch (1995) "excluded results from studies of expert memory, unless the particular expertise was defined by the superior memory performance [...]". This is an important clarification, as their 1995 paper (e.g., pp. 236- 
238) can certainly be interpreted as claiming that LTWM accounts for memory experiments in medical expertise and chess, tasks that do not satisfy EK's definition.

Second, EK (p. 13) note that Ericsson and Kintsch (1995) "were not describing two distinct types of independent mechanisms but rather different types of associations with integrated memory structures in LTM." Again, this is an important clarification. Ericsson and Kintsch (1995) certainly talk about "two different types of encodings" (e.g., p. 221) and the statement that storage in mental abacus calculation is "mediated only by a retrieval structure" (p. 238) seems to imply the two mechanisms are independent in the sense that one can occur without the other. However, I agree with EK that they are dependent in the sense that they act on the same memory structures, in the same way that storage and retrieval are dependent.

The status of the template theory (TT) is another source of disagreement. For EK, TT's scope is limited to memory for chess positions; as LTWM focuses on the move-selection task, direct comparison between the two theories is impossible. However, TT also accounts for data on problem solving (Gobet, 1997, 1998). ${ }^{1}$ In particular, the role of templates in the move-selection task has been formalised in SEARCH, a stochastic model that makes detailed predictions about the macrostructure of search (Gobet, 1997). While SEARCH is not a process model able to play chess, its ability to predict search statistics contradicts EK's claim that TT does not apply to problem solving. Finally, although data on chess problem solving do support the idea of rapid LTM encoding, it is debatable whether they support LTWM, given that LTWM does not incorporate any theory of search or evaluation.

\section{Generic retrieval structures and slotted schemata}

The most theoretically interesting point in this discussion should have centred around the concepts of generic retrieval structures and slotted schemata. Unfortunately, EK ignore key definitions, which makes their argument difficult to follow. My definition of generic retrieval structure (p. 27) clearly allows both slotted schemata (with encoding either of chunks or of individual pieces of information $)^{2}$ and other encoding mechanisms. It also allows information between elements to be encoded — indeed, I used as an example Ericsson and Kintsch's (1995) 64-square retrieval structure, which explicitly has this property. In addition, both Gobet and Simon (1998, p. 228) and Richman et al. (1995, p. 308) specifically state that slotted schemata can store chunks. As a consequence, EK's assertion that generic retrieval structures and templates use slots for storing only individual elements (e.g., digits) is simply incorrect.

One of the motivations behind both EPAM IV and CHREST was to offer computational mechanisms explaining how slotted schemata are gradually acquired and used. EK's radical rejection of this concept ignores two facts: first, that simulations based on slotted schemata account for human data very well, both qualitatively and 
quantitatively, in the digit-span task and in chess. Second, that the concept of schema, in spite of its crucial role in LTWM, is never defined in that theory.

\section{Memory and expertise}

EK raise doubts about the link between superior memory performance and expert performance, and in particular about the time needed to build memory. Two important points should be made here. First, experts learn much more than chunks; in particular, they learn templates, as well as links between chunks and potential actions (Richman et al., 1996). Second, Ericsson and Harris' (1990) novice learned only a small subset of experts' knowledge, but, in compensation, used associations made possible by realworld knowledge that took years to acquire. These points are also relevant to the digitspan task. While both DD and SF drastically improved in less than 100 hours of practice, they drew upon their massive knowledge of running times, dates, etc., which took years to develop.

These ideas are incorporated in Richman et al.'s (1995) detailed simulations of DD using EPAM IV. However, EK criticise this work on two grounds: the simulations do not prove that the retrieval structure in EPAM IV is the only mechanism that could account for the data, and there is no simulation of the experiments carried out parallel to the first 300 practice sessions. What the simulations show is that EPAM IV is sufficient to produce DD's behaviour - something that is way beyond the informal descriptions of LTWM. As for EK's second criticism, it is unfounded. Richman et al. simulated in detail DD's behaviour during 850 sessions, including the first 300, and then successfully simulated various variables from several experiments, such as percentage correct, rehearsal times and pauses - accounting for the data far more extensively and precisely than any other theory.

DD's simulation raises the question of individual differences, which, according to EK, limit the application of computer modelling techniques. I disagree with this conclusion. By using information about the training sequence and behaviour in different tasks, modelling makes it possible to simulate individual differences in detail, something that is not feasible using informal theorising.

\section{Conclusion}

It is likely that the current controversy will be fruitful in inspiring experiments exploring the role of retrieval structure, templates, and retrieval cues in experts' behaviour. As the two approaches share many assumptions, this empirical work will be beneficial for both, as well as for the field at large. Recent attempts to formalise aspects of LTWM as computer programs (Altman \& John, 1999; Gobet, 2000) augur well for developing testable versions of LTWM in the future, versions which may then be compared at a quantitative level with EPAM/CHREST. 


\section{Reference list}

Altmann, E. M., \& John, B. E. (1999). Episodic indexing: A model of memory for attention events. Cognitive Science, 23, 117-156.

Ericsson, K. A., \& Harris, M. S. (1990, November). Expert chess memory without chess knowledge: A training study. Paper presented at the 31st Annual Meeting of the Psychonomic Society, New Orleans, Louisiana.

Ericsson, K. A., \& Kintsch, W. (1995). Long-term working memory. Psychological Review, 102, 211-245.

Gobet, F. (1997). Roles of pattern recognition and search in expert problem solving. Thinking and Reasoning, 3, 291-313.

Gobet, F. (1998). Chess players' thinking revisited. Swiss Journal of Psychology, 57, $18-32$

Gobet, F. (2000). Long-term working memory: A computational implementation for chess expertise. Proceedings of the 3rd International Conference on Cognitive Modelling. Veenendaal, The Netherlands: Universal Press.

Gobet, F., \& Simon, H. A. (1998). Expert chess memory: Revisiting the chunking hypothesis. Memory, 6, 225-255.

Richman, H. B., Gobet, F., Staszewski, J. J., \& Simon, H. A. (1996). Perceptual and memory processes in the acquisition of expert performance: The EPAM model. In K. A. Ericsson (Ed.), The road to excellence. Mahwah, NJ: Erlbaum.

Richman, H. B., Staszewski, J. J., \& Simon, H. A. (1995). Simulation of expert memory with EPAM IV. Psychological Review, 102, 305-330. 


\section{Footnotes}

\footnotetext{
${ }^{1}$ The scope of TT is currently being extended to language acquisition and expertise in physics and computer programming.

${ }^{2}$ This distinction is actually irrelevant within the EPAM/CHREST framework, as "individual" pieces of information such as digits or pieces are themselves encoded as chunks.
} 